\title{
Revisitation of an old antimalarial to combat Psoriatic Arthritis administering an antidote to Santonin to avoid xanthopsia or maculopathy
}

\author{
Lorenzo Martini' ${ }^{1}$, Piotr Brzezinski²
}

\author{
${ }^{1}$ Department of Pharmaceutical Biotechnologies, University of Siena, Siena, Italy, ${ }^{2}$ Institute of Biology and Environmental \\ Protection, Pomeranian Academy, Slupsk, Poland
}

Corresponding author: Lorenzo Martini, E-mail: martinil163@libero.it

\begin{abstract}
Background: The mechanism of action of antimalarials in the treatment of patients with rheumatoid arthritis is hitherto unknown but is thought to involve changes in antigen presentation or effects on the innate immune system. Material and Methods: Patient with psoriatic artritis after ineffective therapy of three kinds of NSAIDs was used following therapy: chloroquine: $250 \mathrm{mg} /$ day for one entire year; -ibuprofene: $600 \mathrm{mg} /$ day for one entire year; thiamine: $1.6 \mathrm{mg} /$ day. Was analyzed of Disease Activity Score (Das28 using three parameters, excluding the self evaluation, because of the problem of the subjectivity according to the method heralded by Van der Heijde DMFM, van't Hof MA, van Riel PLCM, van de Putte LBA. Results: At the beginning Das28 was 2.4 and at the end of the trial it was 1.00. Conclusions: The antidotes to santonine are limewater, chloral hydrate and thiamine. The antidotes Forecasts a novel re-introduction of this antimalarial in therapy against RA as it reveals a good improvement according to the Das28 method of evaluation of remission of complex symptoms of the same Reumatoid Arthritis.
\end{abstract}

Key words: Chloroquine; Antimalarial; Ibuprofene; Thiamine

\section{INTRODUCTION}

Since the the co-author of this paper had already complained the uncontrolled usage of $\mathrm{TNF} \alpha$ inhibitors (that may result perilous and sometimes letal in peculiar cases for elder) to attempt to combat all kinds of Rheumatoid Arthritis (RA) and referred that this specific phaenomenon is extremely common in Italy where a well organised Mafia exists and numbers many supranational pharmaceutical companies and holdings among the acolytes and too many specialists among the adepts [1-3], the A, who will be the "patient" in this seat, would like to propose, together with his physician, who is the corresponding author, a canonical and ancestral therapy that has been often excluded from the orthodox protocols, because of the ocular toxicity of the main drug employed, that is chloroquine.
Given that the mechanism of action of antimalarials in the treatment of patients with rheumatoid arthritis is hitherto unknown but is thought to involve changes in antigen presentation or effects on the innate immune system [4].

The most important toxicities are on the eyes: corneal deposits, extraocular muscular weakness, loss of accommodation (and sensitivity to light), and a retinopathy that may progress to irreversible visual loss.

The very first complication provoked by chloroquine is xanthopsia [4], the same disease evoked by the prolonged assumption of digoxin or hydrochlorothiazide, but even by santonin (from semen contra extracted from Artemisa cina or absinth from Artemisia absinthium (AA), the former used as helminthic in many underdeveloped countries or in the same lands where

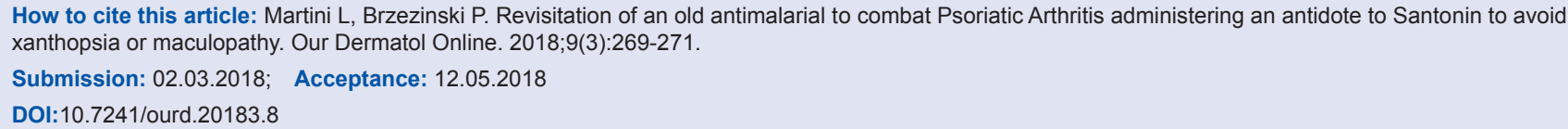


the plant is native to and the latter is used as voluptuary beverage by maniacs and artists) [5].

The proposal of the employ of chloroquine in association with antidotes to semen contra is not peregrin or erratic, the AA deem and are to demonstrate that this hypothesis is not debatable.

\section{MATERIALS AND METHODS}

The report is represented by the co-author himself, who was diagnosed as having seronegative psoriatic artritis three years ago. He presented the typical manifenstations of chronic plaque psoriasis but even other peculiar symptoms as low back pain,conjunctivitis, swollen joints, morning stiffness, foot pain,fatigue and swelling of the toes and fingers.

He underwent to three kinds of NSAIDs, that is: celecoxib for 6 months, niflumic acid for six months, and indomethacin for the residual 6 months.

After 18 months of these types of therapy he, appealing to Sir Thomas Percival's rules on Medical Ethics, has decided to follow the suggestions of his physician (the corresponding Author) and so to self administer the following therapy:

- Chloroquine: $250 \mathrm{mg} /$ day for one entire year,

- Ibuprofene: $600 \mathrm{mg} /$ day for one entire year

- Thiamine: $1.6 \mathrm{mg} /$ day (since the patient drinks generally black tea as voluptuary drink, Equisetum arvense as diuretic and anxiolytics in order to sleep well).

It is well known that some teas, anxiolytics, diuretics and barbiturates can cause the depletion of thiamine,for this the patient made up his mind to increase the daily dosage of vitamin, from $1.2 \mathrm{mg}$ to $1.4 \mathrm{mg}$.

The patient has been avoiding to drink alcohol, smoke cigarettes or eat fresh shell fishes for the entire year of proof.

Thiamine is known to be an excellent antidote to santonin of semen contra since it has beneficial properties on extraocular muscles and on sensitivity to artificial or sun light.

For the sake of truth the patient and the corresponding A are not rheumatologists and for the fact that the coauthor experimented the therapy on himself in corpore vili, he could not examine the observable results (joint pain,joint stiffness at the morning, joint redness and/ or warmth,fatige,fever or weight loss since all of these are subjective remarks).

He could only state the amelioration relying on the Disease Activity Score (Das28 using three parameters, excluding the self evaluation, because of the problem of the subjectivity according to the method heralded by Van der Heijde DMFM, van't Hof MA, van Riel PLCM, van de Putte LBA. in 1993) [6], obtained resolving the following equation:

$0,53938 \sqrt{ }$ (Ritchie's index $)+0,06465(0.01285$, idest the global number of swelling joints/28) +0,330 Ln (VES) +0,00722(GH).

For this, the patient has his own Das28 measured, at the very beginning of the four trials (celecoxib, niflumic acid, indomethacin and finally the synergic combiné chloroquine-anti-inflammatory-thiamine) and recorded all the scores.

All the values revealed after the three prior trials effectuated on himself in corpore vili:

1) Before assuming celecoxib $200 \mathrm{mg}$ twice a day the Das28 was 4.8 and at the end of the proof was 3.9

2) Before assuming niflumic acid $100 \mathrm{mg}$ pro day the Das28 was 3.9 and at the end of the proof was 2.8.

3) Before assuming Indomethacin $200 \mathrm{mg} /$ day the Das28 was 2.8 and at the end of the proof was 2.4.

It is well established that when initial DAS28 value is $>5.1$ one assists at a moderate improvement when the DAS28 score falls down to $>0.6$ at the end of therapy ( 6 or 12 months) and all scores $<1.2$ are not valuable, that is that there has been no improvement in the recovery of illness.

When the initial Das28 is $>3.2$, there is a moderate improvement when after the cure the Das 28 reaches a value comprised between $>0.6$ and 1.2 .

When finally the original Das28 is $<3.2$ there is an excellent improvement when final Das28 will be $>0.6$, a moderate improvement when Das28 is $<1.2$ and no improvement at all when final Das28 is $>1.2$.

\section{RESULTS}

So when the patient and his physician decided to start with the therapy choroquine-ibuprofene-thiamine the co-author's Das 28 was 2.4 and at the end of the trial it was 1.00 . 
The patient had his visual field measured at the beginning of the trial and the scores were 100 degrees laterally, 60 degrees medially, 60 degrees upward, and 75 degrees downward.

The same results were obtained measuring the visual field at the end of the therapy.

No positive scotoma has been observed after 12 months of cure.

\section{DISCUSSIONS}

There is to object that the real value of Das28 at the very beginning of the series of therapies could not be taken for right in this work, as it should be clear that for a net observation of data it would be better if the measure of Das28 should have been effectuated without undergoing to the prior proofs with celecoxib,niflumic acid and Indomethacin.

But all this has been done on purpose, in order to describe a comparison of efficacies among the three common anti-inflammatory drugs and the antimalaric drug.

Knowing that when the difference between the initial score and the final is $>1.2$ the amelioration is valuable, when the difference is $>0.6$ the amelioration is moderate and when indeed is $<0.6$ no valuable improvement can be observed, the A can assert that the combiné chloroquine-anti-inflammatorythiamine shows that the difference of initial and final Das28 is 1.4.

The difference in the case of celecoxib is 0.9 , in the case of niflumic acid is 1.1 and in the case of Indomethacin is 0.4 .

\section{CONCLUSIONS}

Chloroquine has been used for decades in last centuries to try to combat RA, but its employ in medicine was abandoned as the eye toxicity was serious, causing cataract, maculopathy and xanthopsia.

If the therapy with this antimalarial is associated with an antidote to santonin, the biological principle discovered in Artemisia cina by Kahler in 1830 in Düsseldorf, substance that used to evoke irrevocable xanthopsia in teenagers who were forced to assume it as anthelmintic, the eye disorders, it will be demonstrated, will not occur at all.

The antidotes to santonine are limewater, chloral hydrate and thiamine.

The antidotes forecasts a novel re-introduction of this antimalarial in therapy against RA as it reveals a good improvement according to the Das28 method of evaluation of remission of complex symptoms of the same RA.

It must be taken on serious account the problem of xanthopsia or eventual (even rare) problem of maculopathy or cataract.

For this reason a measuration of the visual field is adviced, almost every 12 months.

\section{Statement of Human and Animal Rights}

All procedures followed were in accordance with the ethical standards of the responsible committee on human experimentation (institutional and national) and with the Helsinki Declaration of 1975, as revised in 2008 .

\section{REFERENCES}

1. Martini L. The calvary after the abort due to varied attempts to treat rheumatoid arthirtis by tnf $\alpha$ inhibitors and the final resolution, owing to classic and orthodox remedies in an old lady suffering from a complex syndrome: a special case report. J Pharm Biol Scien. 2017;12: 82-3.

2. Ndiaye M, Ly F, Dioussé P, Diallo M, Diop A, Diatta BA, et al. [The characteristics of severe forms of psoriasis on pigmented skins: A retrospective study of 102 cases in Dakar, Senagal]. Our Dermatol Online. 2017;8:138-42.

3. Tateishi C, Nimmannitya K, Imanishi H, Tsuruta D. Ustekinumab successfully treated a patient with severe psoriasis vulgaris with primary failure to infliximab and secondary failure to adalimumab. Our Dermatol Online. 2015;6:296-8.

4. Restrepo JF, Del Rincon I, Molina E, Battafarano DF, Escalante A. Use of Hydroxychloroquine Is Associated With Improved Lipid Profile in Rheumatoid Arthritis Patients. J Clin Rheumatol. 2017;23:144-8.

5. Martini L. The rediscovery of the Redwood orpiment and a cocktail of plants macerates containing arbutin to defeat the ArribasSilvestre's syndrome in a bien agée upper class lady. Our Dermatol Online. 2017;8:399-401.

6. Van der Heijde DMFM, van't Hof MA, van Riel PLCM, van de Putte LBA. Development of a disease activity score based on judgment in clinical practice by rheumatologists. J Rheumatol 1993;20:579-81.

Copyright by Lorenzo Martini, et al. This is an open-access article distributed under the terms of the Creative Commons Attribution License, which permits unrestricted use, distribution, and reproduction in any medium, provided the original author and source are credited.

Source of Support: Nil, Conflict of Interest: None declared. 